mice. Mice were assessed for weight loss, disease severity, histopathology and endoscopic appearance.

Results We found that during DSS-induced colitis $\operatorname{Pr} d \times 4^{-/-}$mice lost significantly more weight and had a more pronounced disease activity than their wild-type littermates. However, no such differences were observed in $\mathrm{Prdx} 4^{\Delta \mathrm{IEC} / \Delta \mathrm{IEC}}$ mice, compared to their $\operatorname{Prd} x 4^{\text {floxed/floxed }}$ littermates. Likewise, colon histopathology and endoscopy did not reveal significant differences. We next examined Prdx4 expression in dissociated intestinal segments and found that Prdx4 levels in the lamina propria exceeded those of the intestinal epithelium (foldchange $>2$ ). In addition, already under basal conditions, lamina propria immune cell composition differed significantly between $\operatorname{Prdx} 4^{-/-}$and wild-type mice.

Conclusion Our data assign a protective role of Peroxiredoxin-4 in intestinal inflammation which does not arise from the intestinal epithelium but presumably from the lamina propria. Further studies will be needed to determine the functional basis and molecular mechanisms of the observed effects.

Disclosure of Interest None Declared.

\section{PWE-111 HIGHER RED BLOOD CELL METHOTREXATE POLYGLUTAMATES CORRELATE WITH INCREASED DISEASE ACTIVITY, AND ARE USEFUL IN ASSESSING ADHERENCE}

'S Fong*, 'MG Ward, 'I Nasr, 'RM Goel, "KV Patel, 'S Ray, ${ }^{2} \mathrm{M}$ Arenas Hernandez, ${ }^{1} \mathrm{SA}$ Anderson, ${ }^{2} \mathrm{~T}$ Marinaki, ${ }^{1} \mathrm{JD}$ Sanderson, ${ }^{1} \mathrm{PM}$ Irving. ${ }^{1}$ Department of Gastroenterology, GSTS Pathology, Guy's and St Thomas' NHS Foundation Trust, London, UK; 'Purine Research Laboratory, GSTS Pathology, Guy's and St Thomas' NHS Foundation Trust, London, UK

\subsection{6/qutinl-2014-307263.371}

Introduction Methotrexate (MTX) is commonly used in patients with inflammatory bowel disease (IBD). Within red blood cells (RBC), MTX is activated by sequential addition of glutamic acid residues to form polyglutamates (MTXPG ${ }_{1-5}$ ). In rheumatoid arthritis, low [MTXPG] has been associated with active disease, whereas other studies have demonstrated an inverse relationship, including the only published data in IBD. The aim of this study was to determine if RBC [MTXPG] reflect clinical response in IBD patients and whether they are useful in assessing adherence. Methods This was a single-centre, retrospective pilot study of 21 IBD patients treated with weekly MTX. RBC MTXPG $_{1-5}$ was measured using high-performance liquid chromatography. Clinical status (active disease or remission) was assessed by 2 IBD physicians blinded to [MTXPG], using a combination of prospectively recorded clinical activity indices (Simple Colitis Activity Index, Harvey Bradshaw Index), endoscopy, faecal calprotectin and $\mathrm{C}$ reactive protein (CRP). Pearson correlation coefficient, $r$ was calculated to assess the relationship between MTX dose and [MTXPG]. Association between [MTXPG] and clinical response was analysed with unpaired t-test.

Results 4/21(22\%) patients (3 of whom admitted non-adherence) had undetectable MTXPGs and were excluded from further analysis. MTXPG $_{2-4}$ were detected in all adherent patients. $\mathrm{PG}_{3}$ was the predominant polyglutamate accounting for a mean of $43 \%$ of total MTXPG. A linear relationship between dose of MTX and $\mathrm{PG}_{1-5}$ was observed. 12/21(57\%) patients were assessed as having active disease. No significant difference in mean $\left[\mathrm{MTXPG}_{\mathrm{n}}\right]$ was observed between those with active disease and remission. For each $\mathrm{MTXPG}_{\mathrm{n}}$, a non-significant trend towards a higher concentration was observed in patients with active disease.

Conclusion In this study, the largest to date in IBD, measuring RBC MTXPG was useful in assessing adherence to MTX. A trend towards higher PG concentrations was associated with active disease confirming the findings in the only other study in IBD. Whether this is confounded by higher doses being used in patients with more active disease warrants further study in larger, prospective trials.

REFERENCE

Disclosure of Interest: None Declared.

\section{PWE-112 MANAGEMENT OF IRON DEFICIENCY ANAEMIA IN THE OUTPATIENT INFLAMMATORY BOWEL DISEASE COHORT}

S Biswas*, J Simmons, M Myszor, A De Silva. Gastroenterology, Royal Berkshire Hospital NHS Foundation Trust, Reading, UK

\subsection{6/gutjnl-2014-307263.372}

Introduction Iron deficiency is the commonest micronutrient deficiency in IBD and causes impaired quality of life. 35\% of patients have been reported to be iron deficient and $65 \%$ require iron replacement over the course of their disease. We analysed the diagnosis and treatment of iron deficiency in our local IBD cohort and compared this to BSG guidelines. They state that all IBD patients should have an annual full blood count and if anaemic $(\mathrm{Hb}<12 \mathrm{~g} / \mathrm{dl}$ for women, $\mathrm{Hb}<13$ for men) iron studies should be undertaken. If disease is inactive and ferritin $<30$ or there is active disease and ferritin $<100$, the patient should be on iron. This should be the recommended type of iron; IV iron in severe anaemia (haemoglobin $<10$ ) or severe intestinal disease activity, concomitant therapy with an erythropoietic agent, or patient preference. $\mathrm{Hb}$ should be rechecked after 4 weeks and if it does not rise by $2 \mathrm{~g} / \mathrm{dl}$ or normalise, IV iron should be started. If $\mathrm{Hb}<10$ and there is no response to IV iron therapy within 4 weeks EPO should be given.

Methods A prospective study was undertaken in the Royal Berkshire NHS Foundation Trust of patients attending IBD clinics in December 2012. 100 patients attending clinic consecutively were recruited.

\begin{tabular}{|c|c|c|c|c|}
\hline MTX PGn & $\begin{array}{l}\text { Correlation between } \\
\text { MTX dose and MTXPG, r, (p) }\end{array}$ & $\begin{array}{l}\text { Active disease: } \\
\text { [PGn] (nmol/RBC } 8 \times 1012) \text {, mean, SD }\end{array}$ & $\begin{array}{l}\text { Remission [PGn] } \\
(\mathrm{nmol} / \mathrm{RBC} 8 \times 1012) \text { mean, SD }\end{array}$ & $p$ value \\
\hline PG1 & $0.96(p=0.01)$ & $22 \pm 16$ & $15 \pm 1$ & 0.28 \\
\hline PG2 & $0.92(p=0.008)$ & $24 \pm 3.6$ & $17 \pm 2.3$ & 0.17 \\
\hline PG3 & $0.98(p=0.003)$ & $51 \pm 9.8$ & $36 \pm 6.7$ & 0.26 \\
\hline PG4 & $0.94(p=0.019)$ & $19 \pm 4.9$ & $12 \pm 1.7$ & 0.25 \\
\hline PG5 & $0.67(p=0.219)$ & $4.5 \pm 1.5$ & $1.3 \pm 0.73$ & 0.09 \\
\hline
\end{tabular}

